\title{
Senso de humor no envelhecimento: uma revisão de escopo
}

Paulo César Lopes Silva ULSBA, ICS/UCP

Marta Regina Soares de Assunção ICS/UCP

Luís Manuel Mota de Sousa CHRC, UE

| Cristina Maria Alves Marques-Vieira CIIS, ICS/UCP

Helena Maria Guerreiro José UCISA:E, UALG 


\section{RESUMO}

Introdução: O processo de envelhecimento reveste-se de particularidades nas quais o senso de humor parece interferir. Objetivo: Mapear a produção científica sobre os fatores associados ao senso de humor enquanto resposta humana e os seus efeitos nas pessoas idosas. Método: Revisão de escopo alargada segundo a metodologia do Joanna Briggs Institute. Foram pesquisados artigos publicados em português, inglês e espanhol, sem espectro temporal definido nas plataformas Scopus, Web Of Science, CINAHL, MEDLINE, B-ON e Google Acadêmico. Resultados: Foram identificados 228 artigos e incluídos 18 destes. Foi encontrada informação sobre o senso de humor relacionado com a longevidade; a mortalidade; o bem-estar; a saúde; a depressão; a ansiedade; a satisfação com a vida; as relações interpessoais; a qualidade de vida; o enfrentamento; a personalidade; e o gênero. Considerações Finais: $O$ senso de humor é um fenômeno que carece de aprofundamento conceitual. Da análise dos temas emergentes identificados evidencia-se que estes se relacionam entre si e com o senso de humor. Revisão registada na plataforma Open Science Framework (OSF) com o código osf.io/acm89.

Palavras-chave: Senso de Humor e Humor Como Assunto, Saúde do Idoso, Longevidade, Expectativa de Vida. 


\section{- INTRODUÇÃO}

Estudos desenvolvidos na população idosa comprovam que o aumento do cortisol sérico conduz a um aumento do estresse (CASTRO VAZ et al., 2013; SHOLL-FRANCO et al., 2014; SHIMANOE et al., 2021) e, simultaneamente, ocorre uma sobrecarga emocional que apresenta uma forte correlação positiva com o aparecimento de fragilidades e limitações, podendo originar instabilidade psicológica, tendência para o isolamento, e para o aparecimento de síndromes depressivas e distúrbios psicossomáticos (MACENA; HERCULANO; COSTA, 2018). Tais achados levam a refletir sobre fatores ou estratégias que possibilitem aos idosos ultrapassar positivamente o processo de envelhecimento. A promoção do senso de humor parece contribuir favoravelmente na vivência destas alterações comuns no processo de envelhecimento, podendo desempenhar um papel fundamental para a longevidade e um envelhecimento ativo. Esta ideia é suportada por GIBSON (2019) que afirma que o humor pode apresentar-se como um mecanismo de defesa que mantém afastados pensamentos negativos funcionando como promotor do envelhecimento bem-sucedido.

A avaliação da qualidade de vida do idoso reporta frequentemente para o conceito de dignidade humana que, por sua vez, engloba cuidados com a saúde, o culto da sabedoria, o direito ao não sofrimento e, principalmente a busca pelo bem-estar psicossocial e pela felicidade (NETO et al., 2020). Diversos estudos têm destacado estes aspectos e merecem especial atenção, nomeadamente os que abordam práticas que se centram na esfera humana da pessoa idosa e suas relações interpessoais (XAVIER et al., 2020).

O processo de envelhecimento reveste-se de particularidades nas quais o senso de humor parece interferir. Esta variável tem sido amplamente estudada e tem merecido especial atenção nesta população (FECHINE; TROMPIERI, 2015).

Pesquisas na área do humor têm sido desenvolvidas, nos mais diversos contextos, com o intuito de incrementar a qualidade de vida e a longevidade. As suas conclusões permitem justificar a premissa que o humor é benéfico para a saúde e que as pessoas com elevado senso de humor podem ter a perceção de que são mais saudáveis, ainda que, de forma objetiva possam não o ser, para além disto, estas pessoas traduzem menor preocupação com a saúde e sintomas de doença, neste sentido, talvez o humor contribua para uma melhor qualidade de vida, sem tornar fisicamente as pessoas mais saudáveis (MARTIN; FORD, 2018a).

O humor, enquanto intervenção, promove o bem-estar físico e psicológico, o alívio da tensão e fortalecimento do sistema imunitário, incrementa a saúde percebida através da redução de eventos adversos como a dor, o estresse, a ansiedade (SOUSA; JOSÉ, 2016).

Peritos no fenómeno do humor referem que os aspectos emocionais do humor apresentam uma maior expressão no desenvolvimento da competência social do que nos aspectos cognitivos e atitudinais, apontando para evidências de que as competências interpessoais apresentam fortes correlações positivas entre a gestão emocional e a qualidade dos relacionamentos interpessoais (YIP, MARTIN, 2006). 
Espera-se que, no processo de envelhecimento, os idosos inseridos num contexto possam exprimir a sua criatividade e entusiasmo. O senso de humor pode ser a peça fundamental que ajuda a atingir esse objetivo. Os profissionais de saúde e os cuidadores devem estar cientes dos desafios e de como o desenvolvimento do senso de humor pode influenciar o ajuste positivo (DE ALMEIDA; NUNES, 2020), principalmente nesta etapa da vida (MONAHAN, 2014).

\section{- OBJETIVOS}

Identificar a produção científica sobre os fatores associados ao senso de humor enquanto resposta humana e os seus efeitos nas pessoas idosas e identificar os efeitos do humor nas pessoas idosas.

\section{- METODOLOGIA}

A metodologia utilizada foi a revisão de escopo. Estas revisões possibilitam estudar questões gerais e mapear as evidências científicas produzidas por diversas fontes, sendo cada vez mais comum o recurso a esta metodologia (POLLOCK et al., 2021). Ao extrair a essência de um conjunto diversificado de evidências, de forma a atribuir significado e importância a uma determinada área de interesse da disciplina (DAVIS, DREY, GOULD, 2009; POLLOCK et al., 2021), identifica lacunas no conhecimento onde é necessário desenvolver mais estudos de investigação (POLLOCKet al., 2021).

Este tipo de revisão pretende "mapear" a literatura de relevo numa área de interesse, permitindo aos pesquisadores verificar se é necessário realizar uma revisão sistemática. A revisão de escopo partilha várias características com a revisão sistemática uma vez que também segue um método sistemático, transparente e replicável (SOUSA et al., 2018a).

Decorre em cinco etapas: identificação da questão de pesquisa; identificação dos estudos relevantes; seleção de estudos; mapeamento dos dados; e compilação, resumo e relato dos resultados (ARKSEY; O'MALLEY, 2005).

A revisão de escopo seguiu as indicações presentes no JBI Manual for Evidence Synthesis (PETERS et al., 2020) e atendeu às atualizações metodológicas desta técnica de pesquisa (PETERS et al., 2021). Assim, a partir do framework - Paciente (P), Conceito (C [1]) e Contexto (C [2]), isto é, PCC (PETERS et al., 2020), surgiu a seguinte questão de pesquisa: Qual o conhecimento produzido sobre o senso de humor (C [1]) em contexto de saúde (C [2]) nas pessoas idosas $(P)$ ?

A pesquisa eletrônica foi realizada no mês de março de 2021 utilizando as seguintes bases de dados: Scopus (Elsevier) e Web of Science (Clarivate Analytics), e as 
seguintes plataformas: EBSCO Host ${ }^{\circledR}$ (CINAHL Complete e MEDLINE Complete), Biblioteca do Conhecimento Online (BOn) (em todas as suas bases de dados) e Google Acadêmico.

Os descritores utilizados no estudo foram obtidos na plataforma de Descritores em Ciências da Saúde (DeCS). Foram utilizadas outras palavras-chave, além dos descritores.

Foram utilizadas as seguintes equações booleanas:

S1: AB ((Sense of Humor) OR (Wit and Humor) OR (Wit and Humor as Topic));

S2: AB ((Elderly) OR (Aging) OR (Aged) OR (Longevity));

S3: AB ((S1) AND (S2)).

A pesquisa foi conduzida de forma independente por dois pesquisadores da equipe de pesquisa, e a seleção dos estudos seguiu o mesmo método, com a sequência sugerida por diretrizes internacionais atuais (PETERS et al., 2020). Os estudos relevantes foram obtidos de forma faseada, desde a leitura do título até a leitura do texto integral.

Este estudo secundário incluiu artigos cujo texto completo foi possível aceder, publicados nas bases de dados anteriormente referidas e redigidos nos idiomas português, inglês e espanhol.

Os artigos englobados foram estudos primários em torno da população idosa, cujo foco temático fosse o senso de humor, nomeadamente os seus efeitos no processo de envelhecimento.

Estudos que abordavam o humor enquanto intervenção, ainda que na população idosa, não foram incluídos. Não houve limitação temporal na seleção de artigos, considerando o interesse em mapear a evidência produzida e publicada nesta área.

No final do processo de seleção dos artigos houve necessidade de os analisar de forma sistemática. Assim, seguindo a sugestão de (PETERS et al., 2021), coletou-se a seguinte informação: Autor, ano de publicação e país; Objetivos do estudo; população e amostra; métodos/metodologia; tipo de intervenção: resultados e principais achados. Esta informação foi sistematizada numa tabela de forma a possibilitar a organização dos resultados. Seguindo as indicações de PETERS et al. (2020), a revisão foi registada na plataforma Open Science Framework (OSF).

Os artigos incluídos foram classificados de acordo com o nível de evidência considerando as diretrizes JBI Levels of Evidence (JOANNA BRIGGS INSTITUTE, 2021).

Tratando-se de um estudo secundário, cujo desenvolvimento não envolveu diretamente o ser humano, foram garantidos os procedimentos éticos inerentes à investigação, nomeadamente no âmbito do rigor metodológico, na referenciação e respeito pelas ideias dos autores mobilizados. 


\section{- RESULTADOS}

\section{Visão Geral}

Foram identificados um total de 314 artigos. Depois da remoção dos 86 duplicados, foram analisados 228, tendo sido excluídos 194 por leitura de título $(n=165)$ e resumo $(n=29)$.

Posteriormente, foram analisados de forma integral 34 artigos, dos quais 16 foram excluídos. Esta decisão teve em consideração os seguintes motivos: não responder à questão de investigação ou não atender aos critérios de inclusão. Alguns artigos referiam-se ao humor como intervenção, efeitos do humor e estratégias humorosas; consistiam na validação de escalas relacionadas com a personalidade; reportavam revisões narrativas sem referência à metodologia utilizada, reflexões e opiniões; não abrangiam, na sua amostra, pessoas idosas; abordavam a espiritualidade e o envelhecimento positivo; não dispunham de texto integral ou não estavam redigidos em português, inglês ou espanhol. Obteve-se assim, uma amostra de 18 artigos (figura 1).

Figura 1. Prisma Flowchart da seleção de estudos para a revisão de escopo.

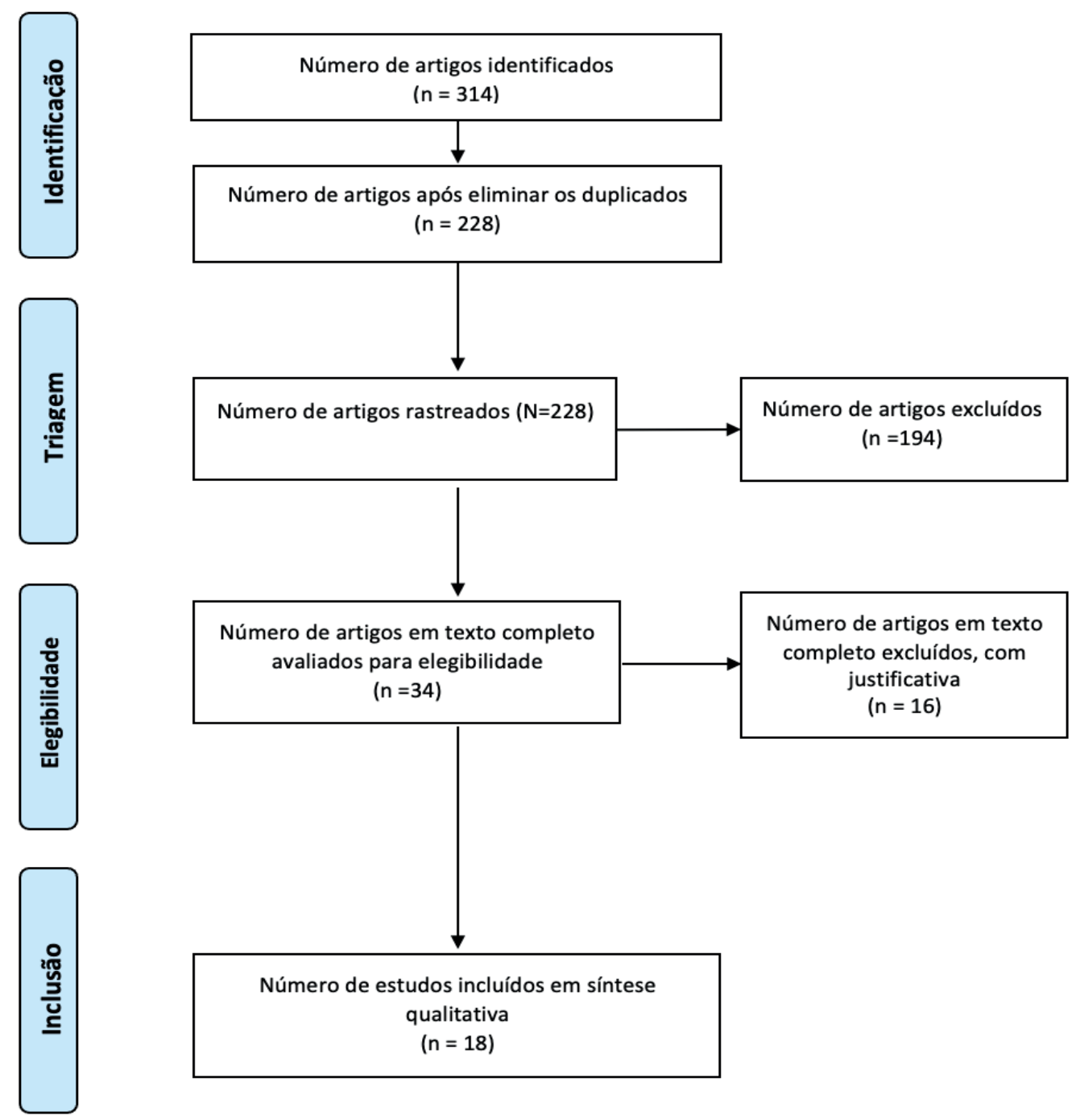


Esta revisão de escopo inclui artigos que recorrem a diversas metodologias. Assim, foram incluídos quatro estudos de natureza qualitativa: dois estudos de caso (LeBLANC, 1987; RYFF, 1989); um descritivo (SCHIAU, 2017), uma teoria fundamentada (LABARCA, 2012) e catorze de natureza quantitativa: nove estudos correlacionais (BOKARIUS et al., 2011; KÖHLER; RUCH, 1996; SVEBACK; MARTIN; HOLMEN, 2004; THORSON; POWELL, 1996; BRAJKOVIĆ et al., 2011; SCHIAU, 2016; THORSTON; POWELL, 1993a; PROYER, RUCH, MÜLLER, 2010; YOUDER, HAUDE, 1995); três longitudinais (LÓPEZ-BENÍTEZ et al., 2017; ROMUNDSTAD et al., 2015; SVEBACK; ROMUNDSTAD; HOLMEN, 2010); um estudo de coorte (THORSTON; POWELL, 1993b); um estudo transversal exploratório (MARZIALI, McDONALD, DONAHUE, 2008).

De acordo com o desenho dos estudos e segundo as diretrizes JBI Levels of Evidence (JOANNA BRIGGS INSTITUTE, 2013), três estudos foram classificados com nível 3e (Oservational study without a control group) (LÓPEZ-BENÍTEZ et al., 2017; ROMUNDSTAD et al., 2015; SVEBACK; ROMUNDSTAD; HOLMEN, 2010); onze com nível 4b (Crosssectional study) (BOKARIUS et al., 2011; KÖHLER; RUCH, 1996; SVEBACK; MARTIN; HOLMEN, 2004; THORSON; POWELL, 1993b; THORSON; POWELL, 1996; BRAJKOVIĆ et al., 2011; SCHIAU, 2016; THORSTON; POWELL, 1993a; PROYER, RUCH, MÜLLER, 2010; YOUDER, HAUDE, 1995; MARZIALI, McDONALD, DONAHUE, 2008) e quatro com nível 4d (Case study) (LeBLANC, 1987; RYFF, 1989; SCHIAU, 2017; LABARCA, 2012)

Quanto ao ano de publicação não se verificou a existência de uma distribuição uniforme, nem houve um ano particularmente expressivo de publicações nesta área (figura 2).

Figura 2. Ano de publicação dos estudos selecionados para a revisão de escopo.

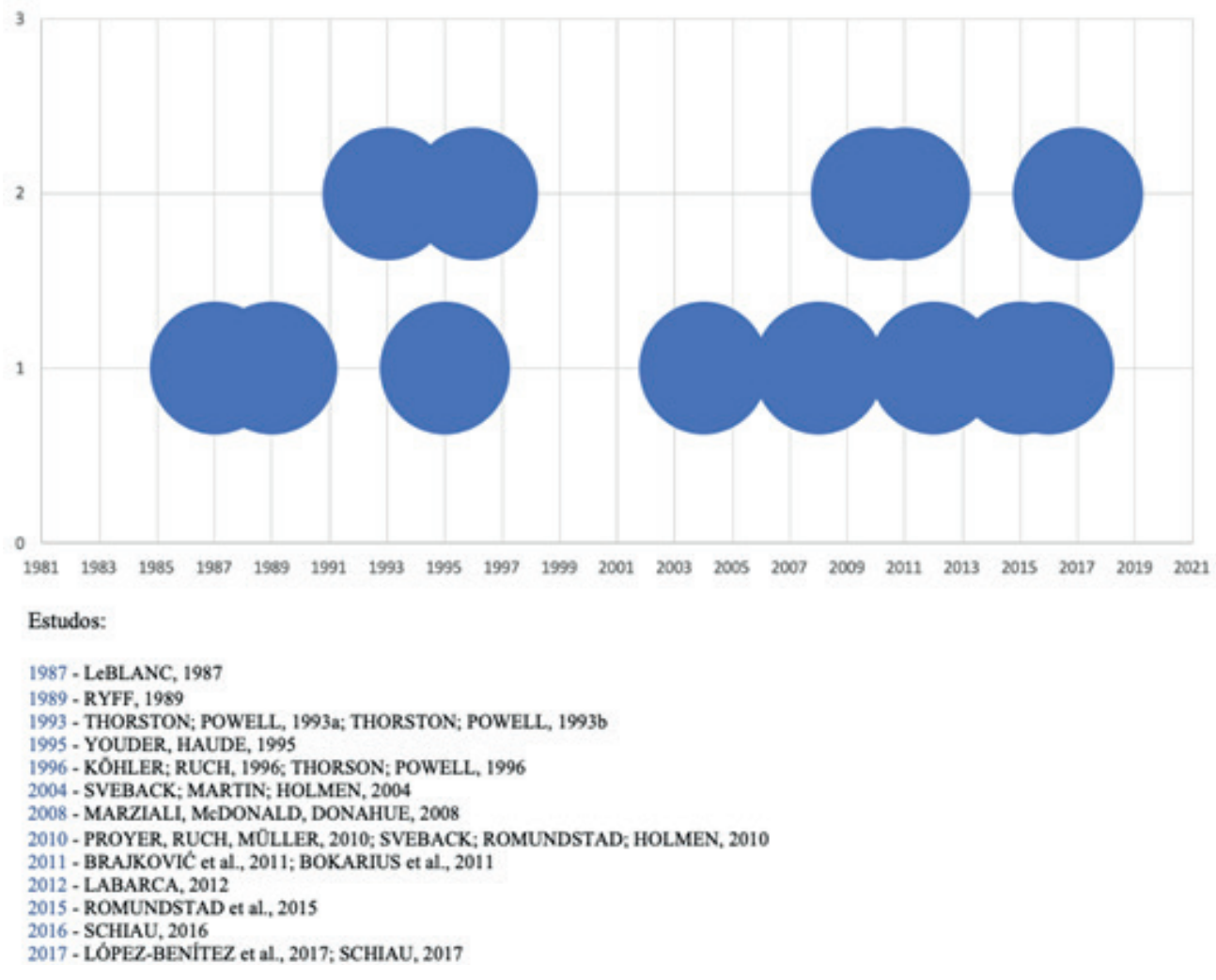


Considerando os países de origem dos estudos, os Estados Unidos da América (EUA) emergem com o maior número de publicações (39\%) (figura 3).

Figura 3. Países de publicação dos estudos selecionados para a revisão de escopo.

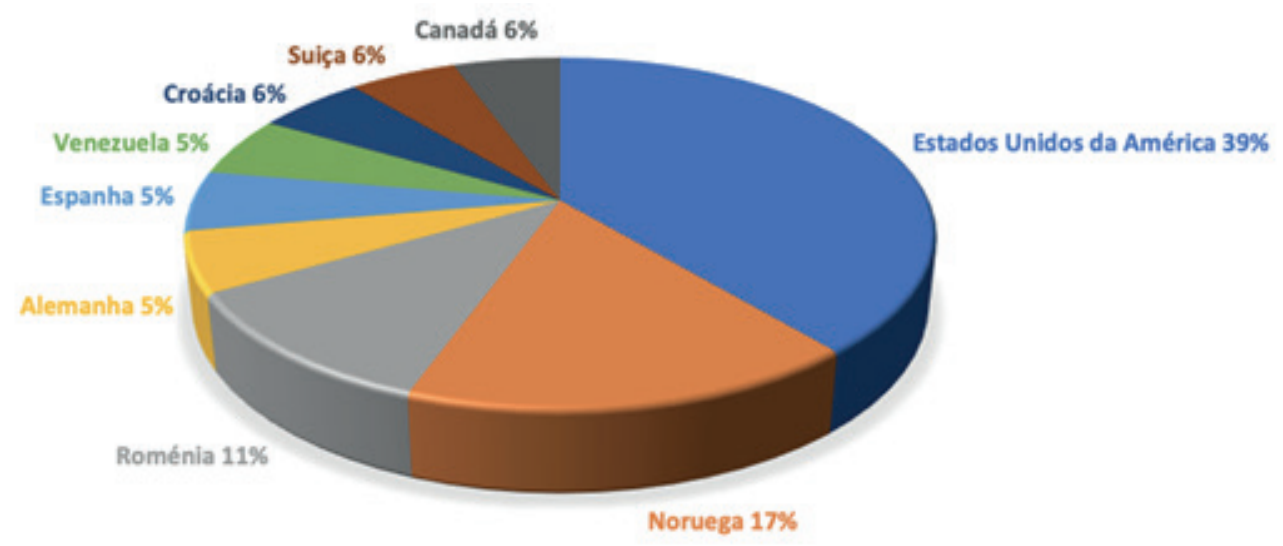

Estudos:

口 BOKARIUS et al., 2011; LeBLANC, 1987; RYFF, 1989; THORSON; POWELL, 1993A; THORSON; POWELL, 1993b; YODER; HAUDE, 1995;

THORSON; POWELL, 1996

Q ROMUNDSTAD et al., 2015; SVEBACK; MARTIN; HOLMEN, 2004; SVEBACK; ROMUNDSTAD; HOLMEN, 2010

口 SCHLAU, 2016; SCHIAU, 2017

D KOHLER; RUCH, 1996

Q LOPÉZ-BENITEZ et al., 2017

口 LABARCA, 2012

BRAJKOVIC et al., 2011

PROYER: RUCH; MULLER, 2010

n MARZIALI; MeDONALD; DONAHUE, 2008

A população em estudo não é homogênea embora todos os estudos abordem a pessoa idosa. Assim, quanto à idade dos participantes: um estudo incluiu participantes acima dos 17 anos (KÖHLER; RUCH, 1996); cinco estudos incluíram participantes acima dos 18 anos (BOKARIUS et al., 2011; LÓPEZ-BENÍTEZ, 2017; THORSTON; POWELL, 1993a; THORSTON; POWELL, 1993b; THORSTON; POWELL, 1996); um estudo incluiu participantes acima dos 19 anos (ROMUNDSTAD et al., 2015); dois estudos incluíram participantes acima dos 20 anos (SVEBACK; MARTIN; HOLMEN, 2004; SVEBACK; ROMUNDSTAD; HOLMEN, 2010); três estudos incluíram participantes acima dos 60 anos de idade (LeBLANC, 1987, LABARCA, 2012; SCHIAU, 2016); seis estudos não especificam a idade dos participantes (RYFF, 1989; BRAJKOVIĆ et al., 2011; SCHIAU, 2017; PROYER; RUCH; MULLER, 2010; YODER; HAUDE, 1995; MARZIALI; McDONALD; DONAHUE, 2008), embora cinco desses estudos refiram que incluem exclusivamente participantes idosos (RYFF, 1989; BRAJKOVIĆ et al., 2011; SCHIAU, 2017; YODER; HAUDE, 1995; MARZIALI; McDONALD; DONAHUE, 2008). Quanto ao gênero, apenas um estudo abordou exclusivamente mulheres idosas (SCHIAU, 2017).

Todos os estudos selecionados exploram o conceito senso de humor. Especificamente, abordam o senso de humor enquanto: disposição para o humor (BOKARIUS et al., 2011), apreciação do humor (THORSON; POWELL, 1993a; KÖHLER; RUCH, 1996) produção do humor (THORSON; POWELL, 1993a; KÖHLER; RUCH, 1996; SCHIAU, 2016; SCHIAU, 
2017; YODER; HAUDE, 1995), uso do humor (SCHIAU, 2016) senso de humor como estado psicológico (LÓPEZ-BENÍTEZ et al., 2017), senso de humor como característica de enfrentamento (MARZIALI; McDONALD; DONAHUE, 2008; THORSON; POWELL, 1993a; RYFF, 1989; THORSON; POWELL, 1993b; THORSON; POWELL, 1996; SCHIAU, 2016), a natureza multidimensional do humor (THORSON; POWELL, 1993a), e senso de humor enquanto qualidade de vida (LABARCA, 2012).

Relativamente ao contexto verificou-se que este é significativamente diverso. Os estudos desenvolveram-se desde um serviço hospitalar (ambulatorial) (BOKARIUS et al., 2011) por comunidade (LeBLANC, 1987; RYFF, 1989; SVEBACK; MARTIN; HOLMEN, 2004; MARZIALI; McDONALD; DONAHUE, 2008; SVEBACK; ROMUNDSTAD; HOLMEN, 2010; BRAJKOVIĆ et al., 2011; LABARCA, 2012; ROMUNDSTAD et al., 2015; SCHIAU, 2017), à universidade (THORSON; POWELL, 1993b; THORSON; POWELL, 1996; LÓPEZ-BENÍTEZ et al., 2017), às residências para idosos (LeBLANC, 1987; BRAJKOVIĆ et al., 2011) e aos clubes séniores (SCHIAU, 2016). De salientar que em alguns estudos, apesar de ser indicada a forma de seleção dos participantes, não é explícito o contexto (THORSON; POWELL, 1993a; KÖHLER; RUCH, 1996; PROYER; RUCH; MULLER, 2010; YODER; HAUDE, 1995).

Da análise qualitativa aos resultados dos estudos incluídos nesta revisão de escopo, emergiram 12 temas interligados com o senso de humor: a depressão; a longevidade; o enfrentamento; a personalidade; a mortalidade; ansiedade da morte; o gênero; a qualidade de vida; a satisfação com a vida; as relações interpessoais; o bem-estar; e a saúde (figura 3).

Figura 4. Temas emergentes da análise qualitativa dos resultados dos estudos incluídos na revisão de escopo

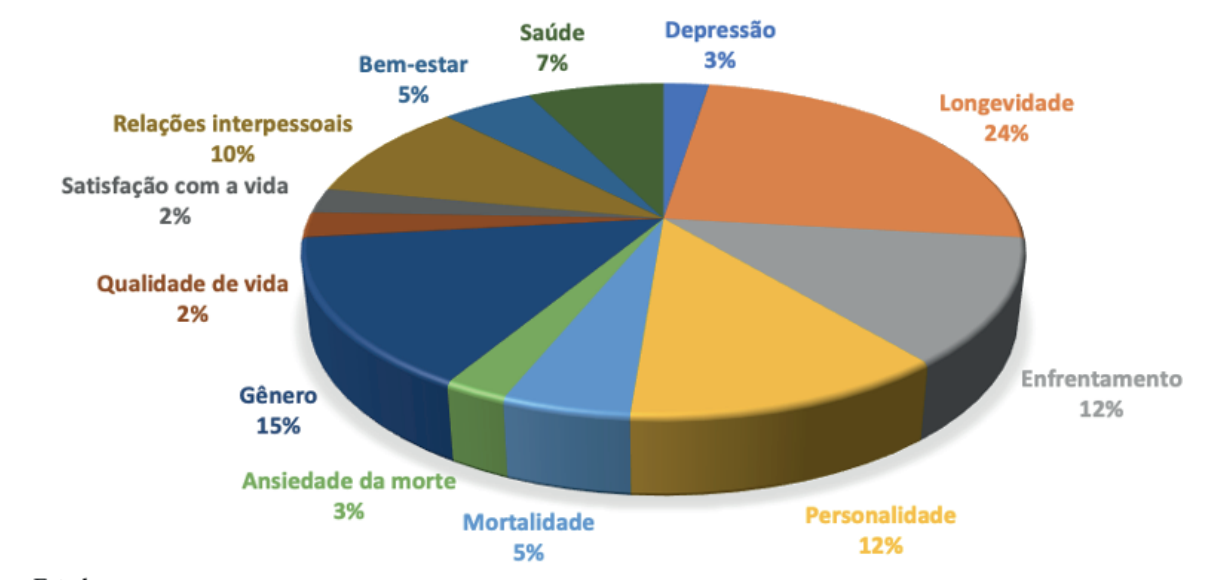

Estudos:

口BOKARIUS et al., 2011

口LEBLANC, 1987; THORSON; POWELL, 1993a; THORSON; POWELL, 1993b; YODER; HAUDE, 1995; THORSON; POWELL, 1996; SVEBACK;

MARTIN; HOLMEN, 2004; PROYER; RUCH; MULLER, 2010; SVEBACK; ROMUNDSTAD; HOLMEN, 2010; LABARCA, 2012; LOPÉZ-

BENITEZ et al., 2017

口LeBLANC, 1987; RYFF, 1989; THORSON; POWELL, 1993a; THORSON; POWELL, 1993b; MARZIALI; MCDONALD; DONAHUE, 2008

口THORSON; POWELL, 1993b; KÖHLER; RUCH, 1996; THORSON; POWELL, 1996; LABARCA, 2012; LOPÉZ-BENÍTEZ et al., 2017

口 SVEBACK; ROMUNDSTAD; HOLMEN, 2010; ROMUNDSTAD et al., 2015

口THORSON; POWELL, 1993a

THORSON; POWELL, 1993a; KÖHLER; RUCH, 1996; THORSON; POWELL, 1996; ROMUNDSTAD et al., 2015; SCHIAU, 2016; SCHIAU, 2017

ILABARCA, 2012

$\square$ BRAJKOVIĆ et al., 2011

I PROYER; RUCH; MULLER, 2010; LABARCA, 2012; SCHIAU, 2016

I RYFF, 1989; PROYER; RUCH; MULLER, 2010

ISVEBACK; MARTIN; HOLMEN, 2004; MARZIALI; McDONALD; DONAHUE, 2008; LABARCA, 2012 


\section{- DISCUSSÃO}

Os estudos selecionados envolvem participantes em diferentes coortes de idade. Embora a maioria dos estudos englobem uma amostra de idosos (LeBLANC, 1987, LABARCA, 2012; SCHIAU, 2016; RYFF, 1989; BRAJKOVIĆ et al., 2011; SCHIAU, 2017; YODER; HAUDE, 1995; MARZIALI; McDONALD; DONAHUE, 2008), outros referem-se a uma amostra mais abrangente de pessoas adultas (inclui idosos e adultos) (KÖHLER; RUCH, 1996; BOKARIUS et al., 2011; LÓPEZ-BENÍTEZ, 2017; THORSTON; POWELL, 1993a; THORSTON; POWELL, 1993b; THORSTON; POWELL, 1996; ROMUNDSTAD et al., 2015; SVEBACK; MARTIN; HOLMEN, 2004; SVEBACK; ROMUNDSTAD; HOLMEN, 2010), o que limita a generalização dos resultados para a população de pessoas idosas (MALLYA; REED; YANG, 2019). Uma amostra mais diversificada é útil para sustentar as conclusões dos estudos no âmbito do fenômeno senso de humor (YIP; MARTIN, 2006; SVEBAK, 2010). Estudos mais recentes centram-se na exploração da correlação entre o senso de humor e o gênero dos participantes (ROMUNDSTAD et al., 2015; SCHIAU, 2016; SCHIAU, 2017).

De acordo com a teoria do senso de humor de SVEBAK (1974), ter senso de humor é demonstrar capacidade de imaginar realidades (fictício) e de se comportar de acordo com as mesmas dentro da sua esfera social (real), de tal forma que esta não seja levada a um estado de ruptura. Sendo um conceito amplamente explorado, a sua natureza multidimensional não permite o alcance de consenso entre a comunidade científica. Trata-se de um conceito multifatorial que atende a aspectos sociais, de desenvolvimento, emocionais, cognitivos e biológicos, que interagem uns com os outros e influenciam o agir humano (GREENGROSS, 2013). Independentemente da idade, o senso de humor parece ser bastante abrangente e permanecer estável ao longo dos grupos etários (PROYER; RUCH; MULLER, 2010). O senso de humor encontra-se positivamente associado ao bem-estar e apresenta-se como uma força de carácter entre os idosos (RUCH; PROYER; WEBER, 2010).

Constatou-se que os estudos se desenvolveram principalmente em contexto comunitário. Este fato é defendido por GEIB (2012) ao afirmar que estudos no âmbito da gerontologia devem desenvolver-se em contextos sociais neutros, onde se minimiza a influência de fatores externos.

No sentido de discutir os resultados da análise qualitativa dos estudos incluídos, e de acordo com as sugestões de POLLOCK et al. (2021), nesta seção abordam-se os temas que emergiram da revisão, nomeadamente a longevidade; a mortalidade; o bem-estar; a saúde; a depressão; a ansiedade; a satisfação com a vida; as relações interpessoais; a qualidade de vida; o enfrentamento; a personalidade e o gênero. 


\section{Temas emergentes da análise qualitativa dos resultados dos estudos incluídos na revisão de escopo}

A longevidade tem sido ambicionada em várias esferas, desde a política à saúde pública. Num estudo os autores encontraram nas expressões dos participantes opiniões contraditórias, ressaltando-se a ideia de que mais tempo de vida era desejável, para alguns, quando fosse possível tê-lo com níveis aceitáveis de saúde, isto é, se a pessoa puder viver de forma independente e não em vulnerabilidade e declínio (EKERDT et al., 2017).

Num estudo com idosos entre os 75 e os 82 anos, os mais velhos consideraram importante ter senso de humor, valorização da vida, espírito resiliente e respeito por si mesmo e pelos outros (LeBLANC, 1987). Os participantes consideraram que ter sentido de humor é essencial para viver. Foi a capacidade de rir e não levar a vida tão a sério que permitiu às pessoas idosas "singrar na vida", assim, ter senso de humor revelou-se essencial para 0 ajuste às mudanças inevitáveis da vida (LeBLANC, 1987).

Também foi verificada uma correlação entre idade e produção de humor, sendo que as pessoas mais velhas mostram menos apreciação do próprio humor e mais apreço por pessoas bem-humoradas (THORSTON; POWELL, 1993a). No entanto, em relação às pontuações na Multidimensional Sense of Humor Scale (MSHS) não foram verificadas diferenças significativas nos diversos quartis etários, mesmo nos indivíduos mais velhos, apesar de diferenças ligeiras, estas não foram estatisticamente significativas (THORSTON; POWELL, 1993b). Mais tarde, com recurso à mesma escala (MSHS) foi verificada uma variabilidade ligeiramente maior nas pontuações dos indivíduos mais velhos, mas não foram encontradas diferenças significativas por idade (THORSTON; POWELL, 1996). Apesar disto, a produção do humor parece aumentar ligeiramente com a idade e os participantes mais velhos mostram uma atitude ligeiramente mais negativa em relação às pessoas bem-humoradas do que os sujeitos mais jovens, mas também indicam atitudes ligeiramente mais positivas em relação ao próprio humor (THORSTON; POWELL, 1996).

Noutro estudo onde também foi aplicada a MSHS, os participantes fizeram as suas autoavaliações e apresentaram as suas estimativas para os seus irmãos falecidos, as avaliações médias nas quatro subescalas da escala aplicada, produziram uma diferença significativa $(p<0,05)$ para a subescala de apreciação de humor, esta associação é contraditória a estudos anteriores e, por isso, a associação positiva verificada entre senso de humor e longevidade, é considerada pelos autores limitada e, entre outros aspectos, os resultados podem ter sido enviesados pelo luto vivido pela perda do irmão e a sua influência nas memórias (YODER; HAUDE, 1995).

Num estudo desenvolvido na Noruega, em 2004, concluem que à medida que as pessoas envelhecem, perdem a capacidade de apreciar, expressar e identificar o humor no 
seu ambiente (SVEBACK; MARTIN; HOLMEN, 2004). As pontuações médias do senso de humor diminuem com o avançar da idade, assim, os participantes com 70, ou mais anos, apresentaram a pontuação mais baixa $(p<0,0004)$. Este decréscimo foi mais pronunciado nas dimensões da apreciação social do humor e na expressividade do riso, do que na dimensão da sensibilidade da mensagem. De acordo com este estudo, pessoas mais velhas tendem a expressar menos alegria e risos, tendem a apreciar menos o humor e, em menor grau, e são menos propensas a notar o humor no ambiente (SVEBACK; MARTIN; HOLMEN, 2004).

Esta ideia também emerge num outro estudo realizado na Noruega, onde foi verificado um ligeiro declínio nas pontuações do senso de humor com o avançar da idade (SVEBACK; ROMUNDSTAD; HOLMEN, 2010). Também na Suíça, ao serem analisadas as habilidades de humor, se verificou que o riso diminuiu com a idade e os mais velhos riem cada vez com menos facilidade. Apesar disto, o grupo de pessoas mais velhas (com mais de 60 anos) obtiveram a pontuação média mais alta no que respeita ao humor positivo (PROYER; RUCH; MULLER, 2010).

O processo de envelhecimento sendo um processo por vezes difícil pode ser uma experiência mais agradável se vivida com humor, o senso de humor é marcado pelas experiências vividas, devendo ser desenvolvido ao longo da vida (LABARCA, 2012). Num estudo mais recente, com uma amostra abrangente e não exclusiva de pessoas idosas, o avançar da idade foi relacionado a mau senso de humor e menor alegria, isto é, o mau senso de humor tendeu a ser ligeiramente superior entre os participantes mais velhos, enquanto que a alegria foi ligeiramente inferior nestes (LOPÉZ-BENÍTEZ et al., 2017).

Recentemente, um estudo etnográfico desenvolvido por FRIEDMAN e FRIEDMAN (2019), demonstra que não existe evidência científica com robustez para concluir que o riso promove a longevidade e a saúde. Esta conclusão vem suportar a inconsistência apresentada nos estudos identificados na presente revisão.

Uma publicação sobre as tendências atuais na expectativa de vida alertou para uma mudança na tendência habitual em 20 países da Organização para a Cooperação e Desenvolvimento Económico (OCDE), verificando-se que o aumento da expectativa de vida nos seis anos mais recentes foi menor do que nos seis anos anteriores e, em alguns países, verificaram-se mesmo diminuições nos últimos seis anos (CRIMMINS, 2021). A contribuir para esta nova tendência está a desaceleração no declínio das taxas de mortalidade por doenças cardiovasculares (CRIMMINS, 2021). CRIMMINS (2021) reforça que para efetivamente prever e projetar a esperança de vida é importante conhecer aprofundadamente como a saúde nos estadios iniciais da vida e na terceira idade estão relacionadas, e de igual forma perceber como é que as alterações nos fatores de risco para doenças crônicas podem 
influenciar as tendências e, ainda, saber como é que as características sociais e biológicas do envelhecimento se conjugam para criar diferenças na expectativa de vida.

A mortalidade associada ao senso de humor é apresentada em dois estudos. Num deles, os participantes com menos de 65 anos e com altos escores de senso de humor reduziram o seu risco de morte em $32 \%$, enquanto que efeitos não significativos de altos escores de senso de humor foram verificados nos participantes com 74 anos ou mais, no entanto, os efeitos do senso de humor sobre a sobrevivência parecem desaparecer após os 75 anos (SVEBACK; ROMUNDSTAD; HOLMEN, 2010).

Num outro estudo a associação entre o senso de humor já foi estabelecida para diferentes causas de morte (ROMUNDSTAD et al., 2015): pontuações elevadas na componente cognitiva do senso de humor foram significativamente associadas a taxas de mortalidade mais baixas em mulheres, o mesmo não se verificou nos homens; a mortalidade por doenças cardiovasculares foi significativamente menor em mulheres com altos escores no componente cognitivo do senso de humor; a mortalidade devido a infeções foi significativamente menor em pessoas com altos escores no componente cognitivo, em ambos os gêneros; os componentes sociais e afetivos do senso de humor não foram associados à mortalidade. Na população total, a associação positiva entre a componente cognitiva do senso de humor e a sobrevivência esteve presente até os 85 anos, a partir desta idade esta associação não se verificou, exceto nas mulheres (ROMUNDSTAD et al., 2015).

De acordo com a Organização Mundial da Saúde, a elevada prevalência de doenças crônicas, multi-morbidade e problemas psicossociais nas pessoas idosas requerem ação, incluindo priorização do bem-estar (WORLD HEALTH ORGANIZATION, 2015a). O bem-estar subjetivo engloba respostas emocionais, domínios de satisfação e o julgamento sobre satisfação global com a vida, sendo composto por afetos agradáveis (como a alegria, o contentamento, o orgulho, o afeto e a felicidade), desagradáveis (como a culpa e a vergonha, a ansiedade e preocupação, a raiva, o estresse e a depressão), satisfação com a vida (como o desejo de mudar, a satisfação com a vida atual, o passado e o futuro) e um domínio de satisfação (como o trabalho, a família, o lazer, a saúde, as finanças e o "eu próprio") (DIENER et al., 1999; SOUSA et al., 2018b).

Dos pontos de vista sobre o envelhecimento positivo emergem padrões que os adultos de meia-idade e mais velhos podem usar para avaliar as suas próprias vidas e, ao mesmo tempo, fornecem caminhos para desafiar e ampliar pesquisas no âmbito do bem-estar. Neste estudo foram destacadas dimensões do bem-estar que foram alvo de pouca atenção na pesquisa empírica e teórica anterior, são elas: a capacidade de aproveitar a vida e ter senso de humor (RYFF, 1989). Uma investigação com métodos mistos (CRACIUN; GELLERT; FLICK, 2017) veio destacar a correlação entre o envelhecimento positivo e o bem-estar, na medida 
em que o aumento de perspectivas positivas sobre o envelhecimento foi associado a um forte aumento no bem-estar, especialmente em grupos vulneráveis, como é o caso dos idosos.

Um estado de espírito lúdico pareceu contribuir para o bem-estar dos idosos. Um escore mais alto de senso de humor em idosos foi associado principalmente à insatisfação com a situação econômica do país, com o governo e a segurança nacional (PROYER; RUCH; MULLER, 2010). Geralmente, o humor foi positivamente associado ao bem-estar pessoal e nacional, no entanto, entre os participantes com mais de 60 anos de idade, o bem-estar nacional (ou seja, satisfação com o governo, segurança ou economia do país) foi negativamente relacionado ao humor (PROYER; RUCH; MULLER, 2010). Estes dados são reforçados por ZINCHENKO et al. (2017) que indicam que o senso de humor traduz uma função social e adaptativa de relevo. Por outro lado, VAN DER WAL e KOK (2019) indicam que gostar de rir ou ter um bom senso de humor não é relevante na aplicação de programas de terapia do riso para a promoção do bem-estar. O simples fato de "rir de nada", por si só, já pode trazer ganhos, e que ter senso de humor pode funcionar como catalisador da implementação de tais programas em terapias de grupo (VAN DER WAL; KOK, 2019).

A saúde é algo considerado fundamental para dar vida aos anos. As pessoas idosas quando questionadas sobre a sua vontade de viver mais tempo, referem maioritariamente que só gostariam de viver mais anos se o seu estado de saúde fosse adequado, esta condição de saúde foi expressada como o desejo de se sentir bem para poder aproveitar a vida, não ser um fardo para outros e com a garantia de capacidade funcional e cognitiva (EKERDT et al., 2017).

Com suporte numa amostra populacional de grandes dimensões, foi possível inferir que não existiu uma forte associação entre o senso de humor e a saúde da população em geral (SVEBACK; MARTIN; HOLMEN, 2004).

No entanto, num estudo posterior, o humor de enfrentamento e a autoeficácia demonstraram ser fatores importantes para explicar o estado de saúde em idosos. As correlações entre humor de enfrentamento, autoeficácia e apoio social sugeriram que o senso de humor pode desempenhar um papel importante no reforço de abordagens autoeficazes para a gestão das questões de saúde, assim, o humor parece ser uma estratégia para lidar com as adversidades da vida comuns no envelhecimento (MARZIALI, McDONALD, DONAHUE, 2008).

A saúde surgiu enquanto componente da qualidade de vida, neste âmbito, ter senso de humor e rir possibilitou aos idosos uma melhor qualidade de vida considerando aspectos como a saúde, a libertação, a autoestima e a gestão de crises vitais (LABARCA, 2012).

A depressão é um fator que se relaciona de forma negativa, com o envelhecimento bem-sucedido. Neste sentido é importante o desenvolvimento de ações que potenciem a 
diminuição da depressão nesta população, através da promoção do senso de humor (GOLJA; DAUGHERTY; KAVCIC, 2020).

O senso de humor encontra-se fortemente correlacionado à depressão nos indivíduos na faixa etária dos 70 a 80 anos, existindo uma correlação negativa entre a pontuação no DTH (Disposition Toward Humor Questionnaire) e a depressão $(r=-0,83)$ (BOKARIUS et al, 2011). Tais valores sugerem que a população mais velha pode ser menos recetiva ao humor no tratamento de síndromes depressivas. Neste estudo em concreto existiram seis participantes nessa faixa etária, onde não se encontrou uma correlação estatisticamente significativa entre o nível de depressão e o senso de humor $(r=-0,22)$, havendo necessidade de confirmar esta tendência com estudos com amostras maiores (BOKARIUS et al, 2011).

De igual forma, observa-se uma tendência para o desenvolvimento do mau senso de humor à medida que a pessoa envelhece, compatível com quadros depressivos. Nesta linha de pensamento, o estudo de LOPÉZ-BENÍTEZ et al. (2017) concluiu que o mau senso de humor teve tendência a ser ligeiramente superior entre os participantes mais velhos, enquanto que a alegria foi ligeiramente inferior nesses mesmos participantes.

A ansiedade relativamente à morte é um fenômeno multidimensional, comum e inevitável na vida das pessoas, sendo transversal em diferentes culturas e religiões (PANDYA; KATHURIA, 2021). Nas pessoas idosas, a ansiedade da morte tem elevada incidência devido a aspectos como: a existência de doenças crônicas, problemas físicos, dependência de outros, vivência de perdas significativas, solidão e a hospitalização, que emerge como um fator preditor deste fenómeno entre os idosos (BIDGOL et al., 2020).

Num estudo que objetivou saber se o senso de humor ajuda a lidar com a crise pessoal da morte, foi verificada uma relação significativa, embora moderada, entre a MSHS e a Revised Death Anxiety Scale (RDAS) ( $r=-0,13, p<0,01)$, isto é, à medida que o senso de humor aumentou, as pontuações na ansiedade da morte diminuíram, esta relação foi mais forte especificamente entre a ansiedade da morte e o humor de enfrentamento, o que significa que aqueles que usaram menos este humor pontuaram mais na ansiedade da morte (THORSTON; POWELL, 1993a). Também foram verificadas correlações negativas significativas entre as pontuações de ansiedade da morte e a apreciação do humor $(r=-0,13, p<0,01)$ e a apreciação de pessoas com senso de humor ( $r=-0,16, p<0,01)$ (THORSTON; POWELL, 1993a).

A importância da satisfação com a vida na população envelhecida é destacada pela premissa "adicionar vida aos anos em vez de anos à vida" (FORSMAN; NORDMYR, 2017). A satisfação com a vida consiste na forma como o indivíduo avalia positivamente sua própria vida, de acordo com critérios determinados por ele próprio (DIENER et al., 1985) e foi reconhecida como um indicador crítico para o envelhecimento bem-sucedido (DUMITRACHE et al., 2018). 
Um estudo correlacional croata (BRAJKOVIĆ et al., 2011), com uma amostra exclusiva de idosos aposentados, observou que: a satisfação com a vida se correlacionou positivamente com o senso de humor ativo $(r=0,24 ; p=0,019)$, mas não mostrou nenhuma correlação significativa com senso de humor passivo e semi-ativo; as variáveis demográficas avaliadas, a autoavaliação do estado de saúde, a autoavaliação da solidão e senso de humor explicaram 52,8\% da variância na satisfação com a vida, sendo que o indicador que se revelou mais significativo foi o senso de humor ativo; a autoavaliação da satisfação com a vida foi positivamente influenciada por viver numa casa de repouso, ter filhos e ter um senso de humor ativo, já uma percepção de saúde pior e a solidão revelaram ter uma influência negativa; por último, um senso de humor ativo contribui para a satisfação com a vida, enquanto a autoavaliação do status de saúde foi menos influente, embora significativa (BRAJKOVIĆ et al., 2011).

No âmbito das relações interpessoais, estudos recentes apontam que a promoção do senso de humor favorece a comunicação, a qual desempenha um papel de relevo nas interações humanas (SOUSA et al., 2019; TORRES-VIGIL et al., 2021) e, no contexto da saúde, o humor, tal como o riso, apresenta-se como uma estratégia terapêutica válida que parece promover a harmonia na relação entre pacientes (ZHAO et al., 2019).

O recurso ao humor revela-se uma componente frequente da interação social, servindo diversos propósitos (PAPOUSEK et al., 2017). Num contexto mais pormenorizado, o escopo dos estudos selecionados clarifica o papel do senso de humor nas interações humanas durante o envelhecimento.

O estudo desenvolvido por PROYER, RUCH e MULLER (2010), na tentativa de encontrar fatores que poderiam contribuir para as experiências de humor na idade avançada, pesquisou as diferenças entre variáveis como ser solteiro versus ter um parceiro; praticar a sua religião versus não praticar a sua religião; nível educacional ou consumo de álcool e nicotina, no entanto, não foram encontradas diferenças significativas. Já face às relações interpessoais, foi verificado que aqueles que mais frequentemente se encontraram com amigos e colegas obtiveram escores mais elevados, nos diferentes aspectos do senso de humor, do que aqueles que se encontram com amigos e colegas com menos frequência (PROYER; RUCH; MULLER, 2010).

Neste sentido, aqueles que frequentemente (mais de uma vez por semana) se encontram com amigos e colegas pontuaram mais alto nas três escalas principais, em comparação com aqueles que não se encontram com amigos (ou que se encontraram menos do que uma vez por semana), ou seja, diversão $(p<0,01)$; humor positivo $(p<0,01)$ e senso de humor $(p<0,05)$. Também foram verificados escores maiores em três habilidades de humor: 
humor verbal $(p<0,05)$, encontrando humor na vida quotidiana $(p<0,05)$ e humor sob estresse $(p<0,05)$ (PROYER; RUCH; MULLER, 2010).

LABARCA (2012) concluiu que os idosos que têm senso de humor e riem podem ter uma melhor qualidade de vida considerando aspectos determinantes nas relações sociais.

Mais recentemente, num estudo que teve como principal questão saber se os adultos mais velhos que relatam valorizar o senso de humor se sentem menos sozinhos (SCHIAU, 2016): os participantes traduziram uma atitude positiva em relação ao uso do humor nas relações interpessoais; quanto mais as mulheres usam o humor em suas interações sociais, menos solidão social sentem ( $r=-0,26, p=0,04)$; na análise de regressão linear foi verificado que as mulheres, dentro do grupo dos "idosos mais jovens" (60-70 anos), beneficiaram do uso de humor nas interações sociais e que, desta forma, o uso do humor contribuiu para uma diminuição da sensação de solidão; foi encontrada ainda uma correlação entre a produção e o uso interpessoal do humor e solidão social para mulheres idosas. Este achado não deve ser, segundo a autora (2016), necessariamente interpretado como uma causa, pois pode significar que o uso do humor no relacionamento ou nas interações interpessoais das mulheres idosas seja porque já experimentaram conexão social e, portanto, uma sensação reduzida de solidão social. No entanto, também pode significar, que as mulheres idosas deste estudo que tiveram conexões sociais podem ainda ter mais oportunidades de usar o humor nessas interações interpessoais (SCHIAU, 2016).

As conclusões apontadas pelos estudos selecionados confluem com as ideias expressadas por PAPOUSEK et al., (2017) que indicam que ter senso de humor é uma característica que se revela importante para a manutenção do vínculo social e, ao mesmo tempo auxilia na formação, na valorização e manutenção das relações interpessoais e intensifica o sentimento proximidade e inclusão.

A qualidade de vida é um conceito subjetivo e multidimensional, podendo ser definido como a percepção do indivíduo da sua posição na vida no contexto cultural e sistema de valores no qual vive, em relação aos seus objetivos, expectativas, padrões e preocupações (WORLD HEALTH ORGANIZATION, 2015b).

O senso de humor relaciona-se com o riso (a sua expressão externa), mas não é sinónimo de rir (LABARCA, 2012). Na tentativa de determinar o significado do senso de humor para a pessoa idosa, num estudo de natureza qualitativa emergiu dos resultados uma categoria central - o senso de humor é qualidade de vida, traduzida por seis categorias subsidiárias: o senso de humor muda positivamente o ambiente, o senso de humor promove o sentimento de pertença, ter senso de humor é um sinal de maturidade, exercer o senso de humor é uma libertação sublimada (LABARCA, 2012). 
Suportados nesta preocupação, diversos investigadores têm abordado a fragilidade em pessoas idosas como um componente que afeta diretamente a qualidade de vida desta população. Num estudo desenvolvido no âmbito da vulnerabilidade social, numa amostra de idosos, foi aplicada a Escala de Fragilidade de Edmonton (JESUS et al., 2018). Esta escala avalia nove domínios, de entre os quais o senso de humor (FLECK, 1999).

Mais recentemente, uma série de estudos clínicos, mostraram que os protocolos de terapia do humor e do riso melhoram significativamente o bem-estar, reduzindo a depressão e a ansiedade e aumentando o humor positivo, a autoestima, e qualidade de vida percebida entre os idosos (MARTIN; FORD, 2018b).

O enfrentamento, nas pessoas idosas, é distinto de outros grupos etários, pois as causas estressoras variam com a idade, neste sentido, as principais perdas associadas ao envelhecimento e à morte envolvem aspectos como a saúde e/ou capacidade física; a funcionalidade; a qualidade das relações emocionais; a morte de entes queridos; a integração social; os bens materiais; a componente financeira; a cognição; a sensação de domínio; a sensação de utilidade, o bem-estar subjetivo e a qualidade de vida (RIBEIRO et al., 2017).

Diversos estudos identificados na presente revisão de escopo exploram a importância do senso de humor para o desenvolvimento do humor de enfrentamento no senescer. Estudos de abordagem qualitativa, desenvolvidos no final da década de oitenta, salientam esse aspecto sublinhando que ter sentido de humor é essencial para o ajuste às mudanças inevitáveis da vida, funcionando como uma estratégia de enfrentamento (LeBLANC, 1987; RYFF, 1989). Tais dados também são destacados num estudo transversal desenvolvido várias décadas depois (MARZIALI; McDONALD; DONAHUE, 2008).

RYFF (1989), com recurso a uma amostra mais diversificada (participantes de meia-idade e idosos), concluiu que ambas as faixas etárias deram ênfase ao crescimento contínuo, ao prazer da vida e ao senso de humor como características essenciais para um enfrentamento eficaz.

Já na década de noventa, dois estudos de abordagem quantitativa descrevem uma forte correlação positiva e significativa entre a idade e o humor de enfrentamento (THORSTON; POWELL, 1993a; THORSTON; POWELL, 1993b) com valores de $p=0,065$ e $p<0.01$ respectivamente. Tais achados são concordantes com um estudo posterior, o qual destaca que pessoas mais velhas recorrem com maior frequência ao humor de enfrentamento do que pessoas mais jovens (THORSTON; POWELL, 1996).

Um estudo mais recente abordou especificamente o humor de enfrentamento (MARZIALI; McDONALD; DONAHUE, 2008), demonstrando que as correlações detetadas entre este tipo de humor, a autoeficácia e apoio social sugerem que o senso de humor pode desempenhar um papel importante no reforço da autogestão na saúde do idoso. Contudo, 
um estudo aponta que o fato de pessoas idosas se apresentarem mais receptivas como participantes, pode representar um viés nos resultados de investigação desta natureza (THORSTON; POWELL, 1996).

A personalidade é um processo que se desenvolve ao longo de toda a vida de forma dinâmica, resultando da interação permanente, ao longo do tempo, de fatores ambientais, genéticos e biológicos. O processo de desenvolvimento da personalidade é individual, assim, apesar de não serem adequadamente conhecidas as diferenças na direção do desenvolvimento da personalidade e na extensão na qual as mudanças ocorrem, a evidência aponta para a continuidade do processo em todas as fases da vida até à idade avançada (ANDREI, 2020).

A personalidade e o senso de humor estão relacionados de diversas maneiras. A Deferência (um dos traços da personalidade) surgiu fortemente correlacionada ao senso de humor e à produção de humor (THORSTON; POWELL, 1993b). Num estudo posterior, estes autores referem que o humor se apresentou como um recurso para reforçar as normas e valores de um grupo, sendo que a personalidade fez toda a diferença, pessoas com maior criatividade e humor demonstraram características de exibição e de domínio nos traços de personalidade (THORSTON; POWELL, 1996).

A personalidade também foi relacionada ao senso de humor por KÖHLER e RUCH que, em 1996, verificaram as seguintes alterações com o avançar da idade: diminuição da iniciação do humor; diminuição nos parâmetros de qualidade da produção do humor; aumento dos traços de personalidade Psicótico e Extrovertido e aumento dos traços de personalidade de Dominância.

Considerando a natureza evolutiva da personalidade já apontada, num estudo de natureza qualitativa, com pessoas idosas, os achados indicam que o senso de humor evolui com pessoas idosas, os achados indicaram que o senso de humor evolui com a personalidade e permite expressá-la (LABARCA, 2012). Já mais recentemente foram apontadas as três bases temperamentais do senso de humor como estado psicológico: a alegria, a seriedade e o mau senso de humor (LOPÉZ-BENÍTEZ et al., 2017).

As diferenças de gênero e o senso de humor têm sido abordados, por exemplo, em estudos desenvolvidos na década de 90 onde foi demonstrado que estas não eram muito significativas (KÖHLER; RUCH, 1996; THORSTON; POWELL, 1993a). Contudo, com a progressão da investigação nesta área, evidenciam-se algumas diferenças.

São relatadas duas diferenças significativas nas subpontuações de humor: as mulheres produzem menos humor do que os homens (THORSTON; POWELL, 1996; SHIAU, 2016) e estas utilizam mais o humor de enfrentamento do que os homens (THORSTON; POWELL, 
1993a; THORSTON; POWELL, 1996). Maiores escores de repulsa ao humor sexual, assim como no humor em geral, foram verificados nas mulheres (KÖHLER; RUCH, 1996).

Apesar disto, num estudo realizado na Roménia, verificaram-se diferenças em relação ao sentido de humor de homens e mulheres contrariando estes resultados (SCHIAU, 2016): os homens relataram usar o humor com mais frequência do que mulheres, em todas as dimensões incluídas no estudo; os homens usaram e produziram mais humor em seus relacionamentos do que as mulheres; os homens relataram usar um pouco mais de humor para adaptação a diferentes situações (humor de enfrentamento). A diferença entre os dois grupos (homens e mulheres) foi pequena e insignificante $(p=0,051)$ : os homens relataram usar mais humor para negociação e propósitos assertivos ( $p=0,04)$; os homens com maior escolaridade foram mais propensos a usar humor adaptativo $(r=0,47, p=0,01)$ (SCHIAU, 2016).

Um estudo longitudinal retrospetivo demonstrou que existiu significância na interação sexo/idade em duas componentes do senso de humor: na componente cognitiva e na componente afetiva, onde as mulheres de idade avançada (superior a 90 anos) tiveram uma pontuação média particularmente alta no humor cognitivo (ROMUNDSTAD et al., 2015). Quanto aos homens, estes relataram mais lacunas na memória do que as mulheres em todas as cortes de idade adulta, sugerindo que a demência pode ser um dos primeiros indicadores de declínio do senso de humor (ROMUNDSTAD et al., 2015).

Um estudo de abordagem qualitativa relata as diferenças de gênero na produção e uso social do humor (SCHIAU, 2017): as mulheres referiram usar a fofoca nas suas conversas humorísticas e consideraram que os homens usam o humor para se gabar; as mulheres tiveram a percepção de que o humor masculino se baseia na afirmação de status, isto porque, acreditam que os homens criam principalmente humor sobre tópicos relacionados com sexo e sexualidade, acrescentam ainda que esse tipo de humor masculino provém de uma crise de masculinidade na vida adulta. Já quanto ao seu humor, as mulheres admitem produzir humor com temática sexual, mas, ao fazê-lo, são "mais delicadas" e não "tão vulgares quanto os homens" (SCHIAU, 2017).

Confluindo com os dados mencionados pelos estudos selecionados, um estudo recente de natureza quantitativa veio demonstrar que não foram detetadas fortes evidências sobre a diferença de gênero na apreciação do humor, mas que, de forma geral, os homens relataram uma maior atração pelo humor agressivo e autodestrutivo, componentes que envolvem produção de humor (LUEVANO et al., 2021).

\section{Limitações e indicações dos autores dos estudos incluídos}

Os artigos selecionados apontam para a necessidade de desenvolver estudos com amostras de maiores dimensões (YODER; HAUDE, 1995; MARZIALI; McDONALD; DONAHUE, 
2008), particularmente aqueles cujos participantes não foram exclusivamente pessoas idosas (BOKARIUS et al., 2011; THORSON; POWELL, 1993b). Deve-se atender ao cálculo do tamanho amostral, pois este confere validade interna ao estudo (BRITO et al., 2016).

As pesquisas devem ser focadas em investigação de natureza teórica e empírica com o intuito de definir de forma mais concreta o construto, identificando claramente os seus componentes, não obstante foi sugerido o recurso a tecnologia mais avançada de construção de instrumentos para mensuração do senso de humor (KÖHLER; RUCH, 1996). A investigação nas diferentes disciplinas de aplicação do senso humor também deve ser aprofundada (KÖHLER; RUCH, 1996). A este respeito, YODER e HAUDE (1995) mencionaram a necessidade da inclusão de outras variáveis, para além das avaliadas na MSHS, nos estudos que procurem apoiar uma associação entre senso de humor e longevidade. E ROMUNDSTAD et al., (2015) sugeriram alteração de três componentes no questionário Sense of Humor Questionnaire (SHQ), nomeadamente, cognitivo, social e afetivo. É neste sentido que são importantes estudos de adaptação cultural destes instrumentos como é o caso do estudo desenvolvido por JOSÉ e PARREIRA (2008) onde se realiza a análise fatorial confirmatória da MSHS numa amostra por acessibilidade e bastante diversificada.

Há necessidade de desenvolver estudos empíricos (nomeadamente estudos longitudinais) sobre o humor entre os idosos (PROYER; RUCH; MULLER, 2010; LÓPEZ-BENÍTEZ et al., 2017) e com mais momentos de avaliação (LÓPEZ-BENÍTEZ et al., 2017). Neste sentido, verificou-se que a maior parte dos estudos foram desenvolvidos com desenhos transversais, podendo as conclusões serem limitadas, por isso, são sugeridas abordagens longitudinais para conclusões mais sólidas (THORSON; POWELL, 1993b). Também são sugeridas abordagens metodológicas que possibilitem observar as diferenças nos tipos de senso de humor usado por homens e mulheres idosos (THORSON; POWELL, 1996; SCHIAU, 2017), bem como comparar grupos com diferentes características étnicas e culturais (THORSON; POWELL, 1993b).

O recurso a instrumentos de recolha de dados, como a entrevista guiada, também foi sugerido, no sentido de possibilitar a obtenção dos significados subjetivos dos participantes sobre o senso de humor e os aspectos que parecem relacionar-se com esta dimensão, proporcionado assim percepções mais específicas sobre o valor das crenças pessoais no enfrentamento (MARZIALI; MCDONALD; DONAHUE, 2008). Esta ideia já era defendida por LeBLANC (1987) que concluiu que a pesquisa sobre as atitudes face aos idosos e ao processo de envelhecimento deveria ser aprofundada com recurso a entrevistas não estruturadas, conduzindo a uma prática mais adaptada. A evolução dos instrumentos de coleta de dados também se espera útil no sentido de caracterizar o tipo de humor que os idosos consideram agradável, e que o mesmo possa ser terapêutico (BOKARIUS et al., 2011). 
Foi sugerido ainda que sejam estudadas as causas de morte, considerando a possibilidade que algumas delas sejam mais influenciadas pelo senso de humor do que outras (SVEBACK; ROMUNDSTAD; HOLMEN, 2010). Em 1989 já havia sido apontado que as relações interpessoais carecem de maior ênfase na investigação no sentido de ser possível a compreensão das principais características do envelhecimento positivo (RYFF, 1989). Futuras investigações devem superar o estudo das percepções, incidindo sobre a comunicação embasada no humor (SCHIAU, 2017).

\section{- CONCLUSÕES}

A análise efetuada às publicações incluídas nesta revisão de escopo, permite concluir que o senso de humor é um fenômeno que carece de aprofundamento conceitual. Apesar de vários estudos englobarem pessoas idosas, a forma como o senso de humor se relaciona com o processo de envelhecimento ainda é pouco evidente e, sendo um fenômeno multidimensional, necessita da inclusão das várias variáveis que o influenciam, que ultrapassam as escalas usadas.

Os temas emergentes nesta revisão de escopo encontram-se relacionados com o senso de humor, no entanto, é evidente que eles próprios relacionam-se entre si.

A realização desta revisão de escopo possibilita a reflexão sobre a avaliação multidimensional da pessoa idosa, nomeadamente no âmbito da esfera emocional e interpessoal, na qual o senso de humor assume um papel relevante. De igual forma, é importante uma abordagem multidisciplinar deste fenômeno para a promoção efetiva e abrangente do enveIhecimento positivo, desde a fase inicial da vida, possibilitando o desenvolvimento do humor nas pessoas e seus familiares.

Observa-se a inclusão de estudos não recentes o que constitui uma limitação da revisão de escopo apresentada. Esse fato aponta, por si só, para a necessidade de desenvolver pesquisas mais recentes nessa linha de investigação.

\section{- REFERÊNCIAS}

1. ANDREI, F. Personality Development. In: CARDUCCI, B.J. et al. (eds) The Wiley Encyclopedia of Personality and Individual Differences: Models and Theories, 2020, p. 335-338, DOI: $10.1002 / 9781118970843 . c h 234$.

2. ARKSEY, H.; O'MALLEY, L. Scoping studies: towards a methodological framework. International journal of social research methodology, v.8., n.1, p.19-32, 2005. DOI: 10.1080/1364557032000119616. 
3. BIDGOL, Z. Z. et al. Death Anxiety and Its Predictors Among Older Adults. Journal of Holistic Nursing And Midwifery, v.30, n.2, p.101-110, 2020. Disponível em: http://hnmj.gums.ac.ir/ article-1-1385-en.html. Acesso em: 23 abr. 2021.

4. BOKARIUS, A. et al., Attitude toward humor in patients experiencing depressive symptoms. Innovations in Clinical Neuroscience, V.8, N.9, p. 20-23, 2011. Disponível em: https://www. ncbi.nlm.nih.gov/pmc/articles/PMC3196329/. Acesso em 4 abr. 2021.

5. BRAJKOVIĆ, L. et al., Life satisfaction in persons of the third age after retirement. Collegium Antropologicum, v.35, n.3, p. 665-671, 2011. Disponível em: https://www.researchgate.net/ publication/51770201_Life_Satisfaction_in_Persons_of_the_Third_Age_after_Retirement. Acesso em: 14 abr. 2021.

6. BRITO, C. J. et al. (2016). Dimensionamento de amostras e o mito dos números mágicos: ponto de vista. Revista Andaluza de Medicina Del Deporte, v.9 n.1, 29-31, 2016. DOI: 10.1016/j. ramd.2015.02.007

7. CRACIUN, C; GELLERT, P.; FLICK, U. Aging in Precarious Circumstances: Do Positive Views on Aging Make a Difference? The Gerontologist, v.57, n.3, p. 517-528, 2017. DOI: 10.1093/ geront/gnv135.

8. CRIMMINS, E. M. Recent trends and increasing differences in life expectancy present opportunities for multidisciplinary research on aging. Nature Aging, v.1, p. 12-13, 2021. Disponível em: https://www.nature.com/articles/s43587-020-00016-0. Acesso em: 22 abr. 2021

9. DAVIS, K.; DREY, N.; GOULD, D. What are scoping studies? A review of the nursing literature. International journal of nursing studies, v.46, n.10, p.1386-1400, 2009. DOI: 10.1016/j. ijnurstu.2009.02.010.

10. DE CASTRO VAZ, F., et al., Cortisol e atividade física: será o estresse um indicador do nível de atividade física espontânea e capacidade física em idosos? Brasília Médica, v.50, n.2, p. 143-152, 2013. Disponível em: https://cdn.publisher.gn1.link/rbm.org.br/pdf/v50n2a09.pdf. Acesso em 14 abr. 2021.

11. DE ALMEIDA, C. V.; NUNES, C. Humor Is Important in Healthcare Relationship? The Perceptions of Doctors and Nurses. Open Access Library Journal, v. 7., n.05, p. 1-16, 2020. DOI: 10.4236/oalib.1106372.

12. DIENER, E. D. et al. The satisfaction with life scale. Journal of Personality Assessment, v.49, n.1, p.71-75, 1985. DOI: 10.1207/s15327752jpa4901_13.

13. DIENER, E. D. et al. Subjective well-being: Three decades of progress. Psychological Bulletin, v.125, n.2, p. 276-302, 1999. Disponível em: https://media.rickhanson.net/Papers/SubjectiveWell-BeingDiener.pdf. Acesso: 25 abr. 2021.

14. DUMITRACHE, C. G.; RUBIO, L.; RUBIO-HERRERA, R. Extroversion, social support and life satisfaction in old age: A mediation model. Aging \& Mental Health, v.22, n.8, pp.1069-1077, 2018. DOI: $10.1080 / 13607863.2017 .1330869$

15. EKERDT, D. et al. Is longevity a value for older adults? Journal of Aging Studies, v.43, p.4652, 2017. DOI: 10.1016/j.jaging.2017.10.002. 
16. FECHINE, B. R. A.; TROMPIERI, N. O processo de envelhecimento: as principais alterações que acontecem com o idoso com o passar dos anos. InterSciencePlace - Revista Científica Internacional, v.1, n.20, 2012. DOI: 10.6020/1679-9844/2007. Acesso em: 24 abr. 2021.

17. FLECK, M. P. D. A. et al. Desenvolvimento da versão em português do instrumento de avaliação de qualidade de vida da OMS (WHOQOL-100). Brazilian Journal of Psychiatry, v.21, n.1, p. 19-28, 1999. Disponível em: https://www.scielo.br/pdf/rbp/v21n1/v21n1a06.pdf. Acesso em: 22 abr. 2021.

18. FRIEDMAN, H. H.; FRIEDMAN L. W. Laughing Matters: When Humor Is Meaningful. Journal of Intercultural Management and Ethics, n.4, p.55-72, 2019. Disponível em: https://papers. ssrn.com/sol3/papers.cfm?abstract_id=3418980. Acesso em: 22 abr. 2021.

19. FORSMAN, A. K.; NORDMYR, J. Psychosocial links between Internet use and mental health in later life: A systematic review of quantitative and qualitative evidence. Journal of Applied Gerontology, v.36, n.12, p.1471-1518, 2017. DOI: 10.1177/0733464815595509.

20. GEIB, L. T. C. Determinantes sociais da saúde do idoso. Ciência \& Saúde Coletiva, v.17, p.123-133, 2012. Disponível em: https://www.scielosp.org/article/csc/2012.v17n1/123-133/. Acesso em: 23 abr. 2021.

21. GIBSON, J. M. Positive psychology. In: GIBSON, J. M. An introduction to the psychology of humor. Nova York: Routledge/Taylor \& Francis Group, 2019. p. 168-184.

22. GOLJA, K.; DAUGHERTY, A. M.; KAVCIC, V. Cognitive reserve and depression predict subjective reports of successful aging. Archives of Gerontology and Geriatrics, v.90, n.104137, p.1-7. 2020. DOI: 10.1016/j.archger.2020.104137.

23. GREENGROSS, G. Humor and aging-a mini-review. Gerontology, v.59, n.5, p. 448-453, 2013. DOI: https://doi.org/10.1159/000351005.

24. JESUS, I. et al. Fragilidade e qualidade de vida de idosos em contexto de vulnerabilidade social. Texto \& Contexto - Enfermagem, v.27, n.4, e4300016, 2018. DOI: https://doi. org/10.1590/0104-07072018004300016

25. JOANNA BRIGGS INSTITUTE. JBI Levels of Evidence, 2013. Disponível em: https://jbi. global/sites/default/files/2019-05/JBI-Levels-of-evidence_2014_0.pdf. Acesso em: 4 abr. 2021

26. JOANNA BRIGGS INSTITUTE. JBI EBP Database Guide. Adelaide: JBI, The University of Adelaide, 2021. Disponível em: http://ospguides.ovid.com/OSPguides/jbidb.htm\#rf. Acesso em: 24 abr. 2021.

27. JOSÉ, H.; PARREIRA, P. Adaptação para Português da Escala Multidimensional do Sentido de Humor (MSHS). Revista Referência, s.II, n.6, p.7-18, 2008. Disponível em: http://www. index-f.com/referencia/2008pdf/060718.pdf. Acesso em: 22 abr. 2021.

28. KÖHLER, G.; RUCH, W. Sources of variance in current sense of humor inventories: How much substance, how much method variance?. Humor, v.9, n 3/4, p. 363-397, 1996. DOI: 10.1515/ humr.1996.9.3-4.363

29. LABARCA, C. M. Sentido del humor en el adulto mayor. Telos: Revista de Estudios Interdisciplinarios en Ciencias Sociales, v.14, n.3, p. 400-414, 2012. Disponível em:http://ojs. urbe.edu/index.php/telos/article/view/2127/1989. Acesso em: 4 abr. 2021 
30. LeBLANC, D. Case studying the philosophy, integrity, and emotional health of the elderly. Educational Gerontology, v.13, n.5, p. 387-402, 1987. DOI: 10.1080/0360127870130502.

31. LÓPEZ-BENÍTEZ, R. et al., Assessing the "humorous temperament": Construction of the facet and standard trait forms of the State-Trait-Cheerfulness-inventory - STCI. Humor-International Journal Of Personality Assessment, v.101, n.1, p.84-95, 2017. DOI: 10.1080/00223891.2017.1368022.

32. LUEVANO, V. X. et al., Attachment as a predictor of attraction to humor styles. Personality and Individual Differences, v. 173, p. 110634, 2021, DOI: 10.1016/j.paid.2021.110634.

33. MACENA, W. G.; HERMANO, L. O.; COSTA, T. C. Alterações fisiológicas decorrentes do envelhecimento. Revista Mosaicum, v. 15, n. 27, p. 223-238, 2018. DOI: 10.26893/rmv15i27.64. Acesso em: 10 abr. 2021.

34. MALLYA, S.; REED, M.; YANG, L. A theoretical framework for using humor to reduce the effects of chronic stress on cognitive function in older adults: An integration of findings and methods from diverse areas of psychology. Humor, v.32, n.1, p.49-71, 2019. DOI:10.1515/humor-2017-0068

35. MARTIN, R. A.; FORD, T. E. The Clinical Psychology of Humor: Humor and Mental Health. In: MARTIN, R. A.; FORD, T (eds.). The psychology of humor, an integrative approach. 2. ed. Londres: Elsevier Inc., 2018b. p.283-318.

36. MARTIN, R. A.; FORD, T. E. The Clinical Psychology of Humor: Humor and Physical Health. In: MARTIN, R. A.; FORD, T (eds.). The psychology of humor, an integrative approach. 2. ed. Londres: Elsevier Inc., 2018a. p.319-336.

37. MARZIALI, E.; McDONALD, L.; DONAHUE, P. The role of coping humor in the physical and mental health of older adults. Aging \& Mental Health, v. 12, n. 6, p. 713-718, 2008. DOI:10.1080/13607860802154374.

38. MONAHAN, K. The use of humor with older adults aging in place. Social Work in Mental Health. v.13, n.1, p.61-69, 2015. DOI: 10.1080/15332985.2014.918922.

39. NETO et al., Envelhecimento ativo: Dignidade e Longevidade com Qualidade de Vida. International Scientific Journal, v.15, n.2, p. 162-176, 2020. Disponível em: http://revista.srvroot. com/isp/index.php/isp/article/view/957. Acesso em: 24 abr. 2021.

40. PANDYA, A-K.; KATHURIA, T. Death Anxiety, Religiosity and Culture: Implications for Therapeutic Process and Future Research. Religions, v.12, n.1, p.1-13, 2021. Disponível em: https:// www.mdpi.com/2077-1444/12/1/61. Acesso em: 23 abr. 2021.

41. PAPOUSEK, I. et al. The Use of Bright and Dark Types of Humour is Rooted in the Brain. Scientific reports, v.7, n.42967, p. 1-8, 2017. DOI: 10.1038/srep42967. Acesso em: 25 abr. 2021.

42. PETERS, M. D. J., et al., Updated methodological guidance for the conduct of scoping reviews. JBI Evidence Synthesis, v.18, n.10, p. 2119-2126, 2020. DOI: 10.11124/JBIES-20-00167.

43. PETERS, M. D. J., et al. Scoping Reviews (2020 version). In: AROMATARIS, E.; MUNN, Z. (Editors). JBI Manual for Evidence Synthesis, JBI, 2020. Disponível em: https://wiki.jbi.global/ display/MANUAL/Chapter+11\%3A+Scoping+reviews. Acesso em: 4 abr. 2021.

44. POLLOCK, D., et al., Undertaking a scoping review: A practical guide for nursing and midwifery students, clinicians, researchers, and academics. Journal of advanced nursing, v. 77, n.4, p.2102-2113, 2021. DOI: 10.1111/jan.14743. Acesso em: 11 abr. 2021. 
45. PROYER, R.T., RUCH, W., MÜLLER, L.Sense of humor among the elderly: Findings using the German version of the SHS. Zeitschrift fur Gerontologie und Geriatrie, v.43, n.1, p. 19-24, 2010. DOI: 10.1007/s00391-009-0082-0.

46. RIBEIRO, M. et al. Estratégias de enfrentamento de idosos frente ao envelhecimento e à morte: revisão integrativa. Revista Brasileira de Geriatria e Gerontologia, v. 20, n. 6, p. 869-877, 2017, DOI: 10.1590/1981-22562017020.170083. Acesso em: 25 abr. 2021

47. ROMUNDSTAD, S. et al., A 15-Year Follow-Up study of sense of humor and causes of mortality: the Nord-Trondelag health study. Psychosomatic Medicine, v.78, n.3, p. 345-353, 2015. DOI: 10.1097/PSY.0000000000000275

48. RUCH, W.; PROYER, R. T.; WEBER, M. Humor as a character strength among the elderly. Theoretical considerations. Zeitschriftfür Gerontologie und Geriatrie, v.43, n.1, p.8-12, 2010. DOI: 10.1007/s00391-009-0080-2.

49. RYFF, C. D. In the eye of the beholder: views of psychological well-being among middle-aged and older adults. Psychology and aging, v. 4, n.2, p. 195-201, 1989. DOI: 10.1037//08827974.4.2.195

50. SCHIAU, I. Humor, loneliness and interpersonal communication: A quantitative study of Romanian older adults. Romanian Journal of Communication and Public Relations, v.18, n.1, p. 89-106, 2016. DOI: 10.21018/rjcpr.2016.1.204.

51. SCHIAU, I. Women gossip and men brag: perceived gender differences in the use of humor by Romanian older women. Română de Comunicare şi Relaţii Publice, v.19, n.1, p.69-76, 2017. DOI: 10.21018/rjcpr.2017.1.231.

52. SHIMANO, C., et al., Perceived stress, depressive symptoms, and cortisol-to-cortisone ratio in spot urine in 6878 older adults. Psychoneuroendocrinology, v.125, p.105125, 2021. DOI: 10.1016/j.psyneuen.2020.105125. Acesso em: 24 abr. 2021.

53. SHOLL-FRANCO, A., et al., Envelhecimento, estresse e sociedade: uma visão psiconeuroendocrinológica. Ciências \& Cognição, v.1, p. 34-53, 2004. Disponível em: http://www.cienciasecognicao.org/pdf/v01/cec_vol_1_m1147.pdf. Acesso em: 19 abr. 2021

54. SOUSA, L. M. M; JOSÉ, H. M. G. Benefícios do humor na saúde: Revisão Sistemática da Literatura. Enformação, v.7, p. 22-32, 2016. Disponível em: https://repositorio-cientifico.essatla.pt/bitstream/20.500.12253/992/1/enformacao_07_2016_Benef\%C3\%ADcios\%20do\%20 humor\%20na\%20saúde.pdf. Acesso em: 21 abr. 2021.

55. SOUSA, L. M. M. et al., Revisões da literatura científica: tipos, métodos e aplicações em enfermagem. Revista Portuguesa de Enfermagem de Reabilitação, Porto, Portugal, v. 1, n. 1, p. 45-54, 2018a. DOI: 10.33194/rper.2018.v1.n1.07.4391. Disponível em: https://rper.aper. pt/index.php/rper/article/view/20. Acesso em: 24 abr. 2021.

56. SOUSA, L. M. M. et al. Subjective Wellbeing Assessment in People with Chronic Kidney Disease Undergoing Hemodialysis. In RATH, T. (ed.). Chronic Kidney Disease - from Pathophysiology to Clinical Improvements. 2018b. p. 281-292 DOI: 10.5772/intechopen.71194

57. SOUSA, L. M. M. et al. Humor intervention in the nurse-patient interaction. Revista Brasileira de Enfermagem, v.72, n.4, p.1078-1085, 2019. Disponível em: 10.1590/0034-7167-2018-0609. Acesso em 25 abr. 2021 
58. SVEBAK, S. The Sense of Humor Questionnaire: Conceptualization and Review of 40 Years of Findings in Empirical Research. Europe's Journal of Psychology, v.6, n.3, p. 288-310, 2010. DOI: 10.5964/ejop.v6i3.218

59. SVEBAK, S. A theory of sense of humor. Scandinavian Journal of Psychology, v.15, n.1, p.99-107, 1974. DOI:10.1111/j.1467-9450.1974.tb00561.x

60. SVEBAK, S.; MARTIN, R. A.; HOLMEN, J. The prevalence of sense of humor in a large, unselected county population in Norway: Relations with age, sex, and some health indicators. Humor-international journal of humor research, v. 17, n. 1-2, p. 121-134, 2004. DOI: 10.1515/ humr.2004.001.

61. SVEBAK, S.; ROMUNDSTAD, S.; HOLMEN, J. A 7-year prospective study of sense of humor and mortality in an adult county population: The hunt-2 study. International Journal of Psychiatry in Medicine, v.40, n.2, p. 125-146, 2010. DOI: 10.2190/PM.40.2.a

62. ROMUNDSTAD, S. et al., A 15-Year Follow-Up Study of Sense of Humor and Causes of Mortality: The Nord-Trøndelag Health Study. Psychosomatic Medicine, v. 78, n. 3, p. 345-353. 2010. DOI: 10.1097/psy.0000000000000275.

63. THORSON, J.A.; POWELL, F.C. Relationships of death anxiety and sense of humor. Psychological reports, 72 (3 Pt 2), p. 1364-1366, 1993a. DOI: 10.2466/pr0.1993.72.3c.1364

64. THORSON, J.A.; POWELL, F.C. Sense of humor and dimensions of personality. Journal of Clinical Psychology, v.9, n.6, p. 799-809, 1993b. DOI: 10.1002/1097-4679(199311)49:6<799::AlD-JCLP2270490607>3.0.CO;2-P

65. THORSON, J.A.; POWELL, F.C. Women, aging, and sense of humor. Humor, v.9, n.2, p. 169186. 1996. DOI: 10.1515/humr.1996.9.2.169.

66. TORRES-VIGIL, I. et al. The role of empathic nursing telephone interventions with advanced cancer patients: A qualitative study. European journal of oncology nursing : the official journal of European Oncology Nursing Society, [s.I.], v.50, p.101863, 2021. DOI: 10.1016/j. ejon.2020.101863. Acesso em: 25 abr. 2021.

67. XAVIER, L. G. S., et al. Conceito de cientificidade na conceituação e reflexões do envelhecimento: narrativas pertinentes. Revista Diálogos Possíveis. v. 19, n. 2, jul/dez. 2020. Disponível em: https://www.researchgate.net/publication/349439577_Conceito_de_cientificidade_na_conceituacao_e_reflexoes_do_envelhecimento_narrativas_pertinentes

68. YIP, J. A.; MARTIN, R. A. Sense of humor, emotional intelligence, and social competence. Journal of research in personality. v.40, n.6, p.1202-1208, 2006. Disponível em: http://www. jeremyyip.org/uploads/1/2/6/7/12676056/yip_martin_2006.pdf. Acesso em: 24 abr. 2021.

69. YODER, M. A.; HAUDE, R. H. Sense of humor and longevity: older adults' self-ratings compared with ratings for deceased siblings. Psychological reports, 76 (3 Pt 1), p. 945-946, 1995. DOI: 10.2466/pr0.1995.76.3.945.

70. VAN DER WAL, C.N.; KOK, R. N. Laughter-inducing therapies: Systematic review and meta-analysis. Social Science \& Medicine, n.232, p.473-488, 2019. DOI: 10.1016/j.socscimed.2019.02.018. 
71. WORLD HEALTH ORGANIZATION. The World Health Organization quality of life assessment (WHOQOL): Position paper from the World Health Organization. Social Science \& Medicine, v.41, n.10, p.1403-1409, 2015b. DOI: 10.1016/0277-9536(95)00112-k

72. WORLD HEALTH ORGANIZATION. World report on ageing and health. 2015a. Disponível em: http://apps.who.int/iris/bitstream/handle/10665/186463/9789240694811_eng.pdf;jsessionid=7609D6C7218136BCDEDE3DC09523B35A? sequence=1. Acesso em: 23 abr. 2021.

73. ZHAO, J. et al. Effect of humour intervention programme on depression, anxiety, subjective well-being, cognitive function and sleep quality in Chinese nursing home residents. Journal of Advanced Nursing (John Wiley \& amp; Sons, Inc.), [s. I.], v.76, n.10, p. 2709-2718, 2020. DOI 10.1111/jan.14472. Acesso em: 25 abr. 2021. 